www.conferenceie.ase.ro

\title{
AGGREGATING AN OPTIMIZED MANAGEMENT OF HEALTHCARE DATA FROM AN IOT-ENABLED SMART ENVIRONMENT WITH VIRTUAL MACHINES AND CLOUD COMPUTING. A CASE STUDY
}

\author{
Dragoș Nicolae NICOLAU \\ National Institute for Research and Development in Informatics \\ dragoș.nicolau@ici.ro \\ Adriana ALEXANDRU \\ National Institute for Research and Development in Informatics \\ adriana.alexandru@ici.ro \\ Marilena IANCULESCU \\ National Institute for Research and Development in Informatics \\ marilena.ianculescu@ici.ro
}

\begin{abstract}
The global population is ageing at a sharp pace. Elderly is particularly associated with an increased need for social and medical assistance, home care and nursing houses. The existence of complete and correct information is critical to assessing the health condition of seniors, improving well-being and health-related quality of life. Health monitoring systems in smart environments become a viable alternative to traditional healthcare solutions. This paper aims to highlight the opportunities brought by an Internet of Things-enabled smart environment, cloud computing and virtual machines for a proper management of healthcare data. The ongoing project RO-SmartAgeing is presented as a case study for how these technologies can be rightsized in order to increase efficiency and the response time in the remote monitoring of a senior.
\end{abstract}

Keywords: virtual machine, IoT, healthcare data, smart environment, cloud computing, senior patient

JEL classification: $\mathrm{I} 12, \mathrm{I} 31, \mathrm{O} 33$.

DOI: $10.12948 / \mathrm{ie} 2019.04 .18$

\section{Introduction}

The worldwide population is broadly ageing due to the decline of, improvements of healthcare services and policies, advancements in health domain and continuously emerging technology. "The EU-28's population is projected to increase to a 528.6 million around 2050 and thereafter gradually decline to 518.8 million by 2080. [1]. By 2050, the number of people aged 65 or older (considered as elderly people) is expected to be about 1.5 billion, representing 16 percent of the world's population. [2].The current ageing society comes both with challenges and opportunities. Among the main challenges are: a decreased labor force participation, higher expenditures of healthcare and social systems, lack of ageing-specialized caretakers, appropriate age-friendly environments etc. Nevertheless, a diversity of opportunities come along with ageing: implementing new models and patterns for an independent, active and healthy living, personalized medicine and therapeutical protocols, broadly developing smart cities, communities and technologies, citizens' empowerment.

Older population implies a demand for transforming the healthcare services from reactive to proactive ones, with an age-friendly, elderly patient-centered and personalized approach, 
focused towards prevention and avoiding hospitalization and institutionalization in nursinghomes as much as it is benefic for the senior and its family.

Smart environments represent one important mean to provide a dignified and qualitative life of the elderly. A smart environment should be able to ensure a safe, personalized and secure home in which the daily activities and health status parameters of the senior, together with the ambiental parameters can non-intrusively be monitored in order to set a triggered alarm in case of the occurrence of a (potential) harmful event or for a customized healthcare management. In this respect, the remote monitoring can be operated at several scales: at the individual level (aiming to provide e-services for supporting an independent and healthy life of the senior under a customized supervision of health specialists), at the group level (incorporating persons with common interests and goals in health monitoring applications) and at the community level (aiming to use collective data analytics gathered from a very large number of people in their benefit) [3].

The specific technologies associated to a smart environment (such as Internet of Things, Cloud computing, Virtual Machines, etc.) are introduced in chapter 2 of the paper.

A proposed conceptual solution for an optimized management of healthcare data, with a focus on the aggregation among IoT, virtual machines and cloud computing capabilities is presented in chapter 3. The solution is intended to be developed inside the ongoing RO-SmartAgeing project which is briefly reviewed.

\section{Smart environments enabling smart services for senior patients}

\subsection{Senior patients, a high-need patient group}

Population aging is the main social transformation all over the world.

In many advanced countries, various problems caused by this fact have appeared, such as economic problems related to reduced productivity or increased medical and care costs, and social welfare problems related to old people care and social security. Besides, the social participation, leisure and lifelong learning needs of the seniors would have been taken into account. Thus, due to the global ageing, there are growing demands for material and financial resources in health and social systems. The provision of smart health services would enable older adults to be permanently in touch with the medical professionals or caregivers satisfying the growing accountability to their own health.

\subsection{Smart environments for senior patients}

Taking into consideration the global aging of population, it has become necessary to clearly identify the specific seniors' health problems, needs, living conditions and life experiences, and to provide real time communication between health care professionals and patients.

\section{Distinctive performances/features}

Challenges generated by the global ageing of population enforce distinctive performances to be offered in healthcare focused on senior patients. Here are some of the most important ones: Emergence and rapid development of intelligent products aimed at improving the life quality of the elderly users, caused by the development of science and technology; Ability to achieve better access to care, increased treatment options, and the creation of new opportunities for personalized and patient-centered medical acts; Provision of new ways to deliver personalized health care and health care services; Migration to the patient-centered healthcare by monitoring and integrating patient's medical data collected in and out of a clinical environment in treatment protocols, clinical research studies, remote monitoring programs, and preventive actions and measures; Continuing surveillance of the physical signs of older adults with worse age-related health status; Enabling users to receive immediate feedback on their physical 
conditions anywhere and anytime due to the advancement of health information technologies; Reduction of physical check-up cost and decreasing the risk of hospital admission by using wearable systems at home; Facilitating the healthcare delivery process and mitigating the social burden by ubiquitous usage of smart wearable systems for older adults; Providing costefficient adapted (and increasingly complex) care services in senior residences.

\section{Specific technologies}

The request of the ageing population for healthcare services and assistance is increasing. The convergence between innovative digital devices and technologies and health care services provides real-time gathering of medical / environmental data and information, and the availability of massive amounts of patient-related data.

Older people have increasingly adopted digital technologies, facing different types of physical, psychological and social challenges. The acceptance of digital technologies in everyday life is influenced by the variation of degenerative disorders or technological literacy and background [4].

The Internet of Things (IoT) is a recent paradigm related to the interconnection of a huge amount of heterogeneous objects (things) through the Internet. This is granted by the latest technological advances in smart sensors, communication, and Internet protocols. In healthcare, IoT reduces the risks of human errors during data acquisition by using the automatic medical data collection method. This leads to the improvement of the quality of the diagnosis and to the reduction of the risk of human errors during gathering or transmission of false information, which could have a negative impact on the patients' health. The application of IoT in healthcare is divided into [5]: Clinical care: sensors are used to collect physiological information, which is analysed for identifying emergency condition for risky patients, stored on the cloud and sent via Internet to caregivers (patient's family members, nurse) for further analysis; Remote monitoring: is useful for elderly, children or people with chronic illnesses (such as cardiopulmonary disease, Asthma, and Heart failure), which are located far away from medical care facilities. It help caregivers to have real-time pre-diagnosis and earlier intervention before things go wrong; Context-awareness: provides the ability to find the patient's condition and the environment where the patient was located by using different types of sensors to acquire various information about the patient's physical condition (such as his walking, running, sleeping etc.). Such information is helpful in emergency cases for patient' location and for being aware of the type of recommended emergency intervention.

IoT computing aims at automated extraction of data from Internet-connected things and at controlling them in a more informed fashion. [6].

Wireless Sensor Networks (WSNs) is another related technology. Such a network consists of interconnected sensors, each equipped with a processor and a flash memory, and can be interfaced to a computer via a gateway [7].

Smart wearable health monitoring technologies are used to detect deteriorations in cognitive ability, mobility, and psychosocial functioning of older adults [8]. Physical condition data are transmitted, through wireless sensor networks, to a medical center for providing real-time healthcare to patients.

A smart wearable system (supported by technologies, such as wireless sensor networks and electronic care monitoring devices) can be implemented by using wearable sensors (smart clothes, implantable devices, skin devices, and other wearable gadgets), actuators, and smart devices for health evaluation and decision support. Such systems mainly work in monitoring vital signs, body movement, location, and fall prevention [9], [10].

"Cloud computing is a computing paradigm for providing anything as a service such that the services are virtualized, pooled, shared, and can be provisioned and released rapidly with 
minimal management effort. For the users, the services can be accessed conveniently, ubiquitously, across the network, dynamically, and on demand; can be configured with minimal interaction with the service provider; and are elastic and metered on a pay-per-use basis" [11]. Mobile computing is a computing paradigm applicable to all programmable, portable, wireless, and conveniently held devices including, but not limited to, smartphones, tablets, pads, smartwatches and laptops that can be used anywhere and at any time. Most m-health applications are intended for healthcare professionals for disease diagnosis, drug reference and medical calculations [12]. Mobiles and especially smartphones are also considered as packages of sensors and hardware features from which context can be extracted.

The continuous up growth of virtualization technology has made Virtual Machines (VM) turn out into a usual way of providing specific tasks which are distinct from the ones performed in the host environment. A VM is the core of the virtualization and it is basically an emulated computer system using specific software and hardware. It aims to allow the users to perform other tasks than the ones restricted by the physical characteristics of a server. A VM keeps going to use the physical system resources (like RAM, CPU, HDD) while it is distinct and apart from the other installed software. Therefore, a VM can be built, transformed, or cancelled with no impact on the host. A VM behaves as the actual hardware and it is perceived as a physical computer. While in the real world a unique application and a single operating system use the physical resources, in a virtual architecture there might be several VMs running simultaneously on a single server through a software layer - hypervisor - interposed between the hardware and VM. The VMs can be installed in the cloud server.

Among the main advantages of VMs are: a single physical server can host (through hypervisors) multiple VMs with different applications running on, eliminating the need of inefficiently exploited PCs [13]; easy accessibility, scalability, management and maintenance; alternatives for recovery or replacements in case of disasters.

In healthcare VMs have been increasingly applied because of the growing usage of cloud computing in eHealth, of the need for a better management of the health-related data gathered through IoT, of the smart devices and remote monitoring technology

\section{RO-SmartAgeing project, a framework for an optimal management of healthcare data 3.1 Brief presentation of RO-SmartAgeing}

"Non-invasive monitoring and health assessment of the elderly in a smart environment (ROSmartAgeing)" is an is an ongoing project whose outcomes will be able to support the provision of more advanced elderly-centered healthcare services by facilitating the orientation of medical practice to prevention and personalized assistance.

In this context, it is developed a system for monitoring and assessing the state of health of the elderly, integrating non-invasive motion and physiological sensors for the collection of health parameters, a smart environment with discreet environmental sensors to monitor daily activities and lifestyle and a cloud platform for storing and aggregating data, including the presentation of the results of advanced data analysis.

\subsection{A proposed conceptual solution}

RO-SmartAgeing, as a multi-dimensional remote health Monitoring System based on IoT devices, is confronted with the need for real-time processing of a rapidly increasing volume of data, as physiological and environmental parameters are measured at a high rate (at relatively short intervals of time).

In order to monitor a small number of patients, the computing resources provided by a single virtual machine (hosting the Server component and working in the cloud) offer very good satisfaction. Things, however, change dramatically if it is necessary to monitor a high number 
of patients. In this situation, it becomes mandatory to increase the computing capacity by migrating, on the server, from the "single-virtual machine" to a "multi-virtual machine" system. Cloud computing is the best method to host the Server component of an eHealth system because it offers:

- Extremely generous and easily configurable storage capacity;

- Possibility of establishing extremely fast communication between computing units (machines).

The conceptual solution that the present paper proposes is mainly concerned with the fructification of very fast physical communication between the computing units.

Computing Units are web services running each under its own virtual machine. It must be underlined again that a virtual machine is an operating system that runs under the governing of a host operating system.

RO-SmartAgeing System is intended to manage two types of data streams: the first flow consists of structured medical data provided by the data gathering devices, the second flow consists of information resulting from data processing. Attached to these streams, there are the above-mentioned computing units (Web services), that perform the following types of role:

- Decrypting, decoding and separating the piece of information currently received from the IoT medical device; re-encrypting the components thus obtained, followed by their inserting into the database as a new record (the useful information will contain the patient's name, the unique identifier, the name of the measured medical parameter, the value of the medical parameter, the time and the day);

- Upon the arrival of a request - decrypting and processing a chosen medical parameter of a certain patient (drawing a graph of the parameter as a function of time, issuing an alarm notification, etc.).

Figure 1 shows the functional scheme for gathering and sending medical parameters. In order to monitor the patient, the patient medical data are collected by specialized IoT sensors and transmitted using wireless network to the cloud resident server.

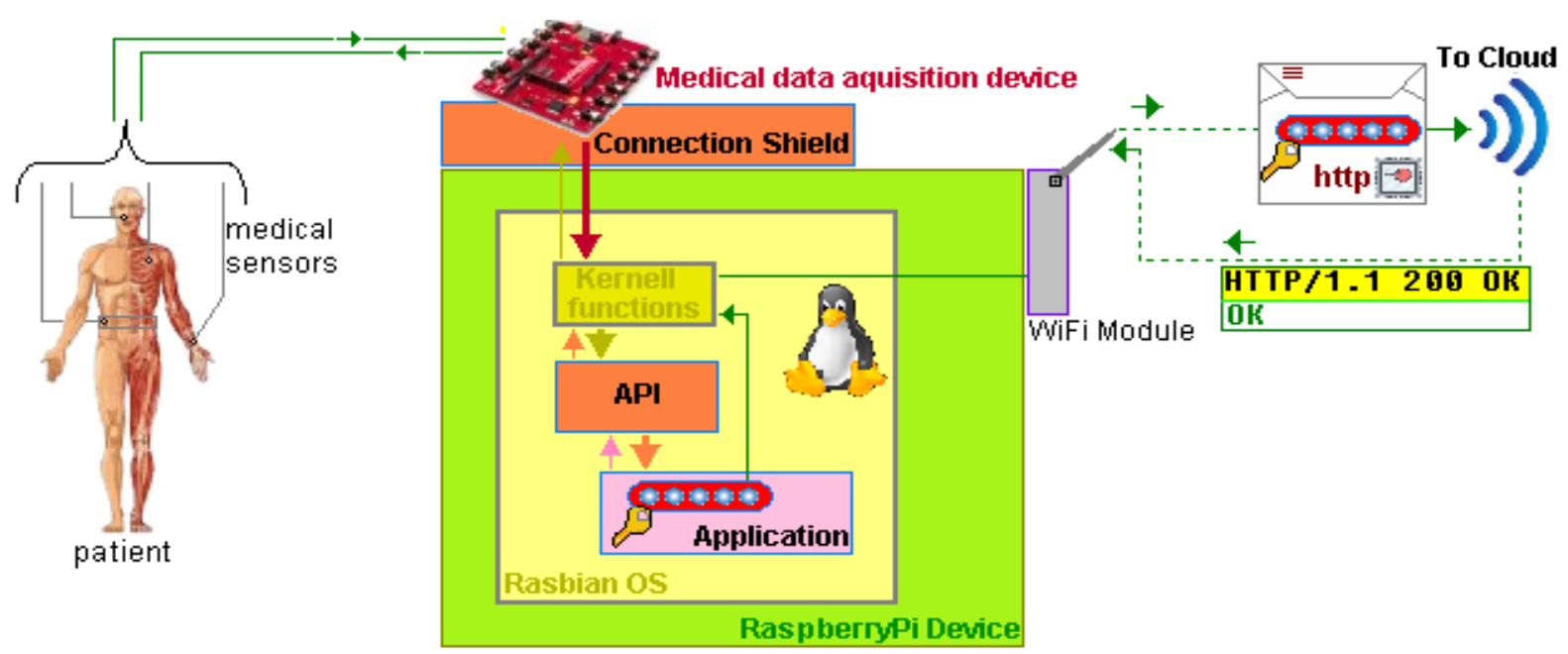

Figure 1. Gathering and sending medical data to the Cloud resident Server

Two types of sensors: medical (main sensors), "attached" to the patient in order to measure vital parameters, and environmental (secondary sensors), placed in the room where the patient is located. The values of the medical parameters define patient's real-time health status, being immediately packed inside the HTTP-Post request and transmitted to the server-side 
component of the platform where processing is performed when asked for by remote client application(s).

The proposed conceptual solution aims at optimizing the response time offered by the server component of the system under the constraint of an intensive flow of requests for data processing and real time responses.

In Figure 2 is represented the server-side proposed solution.

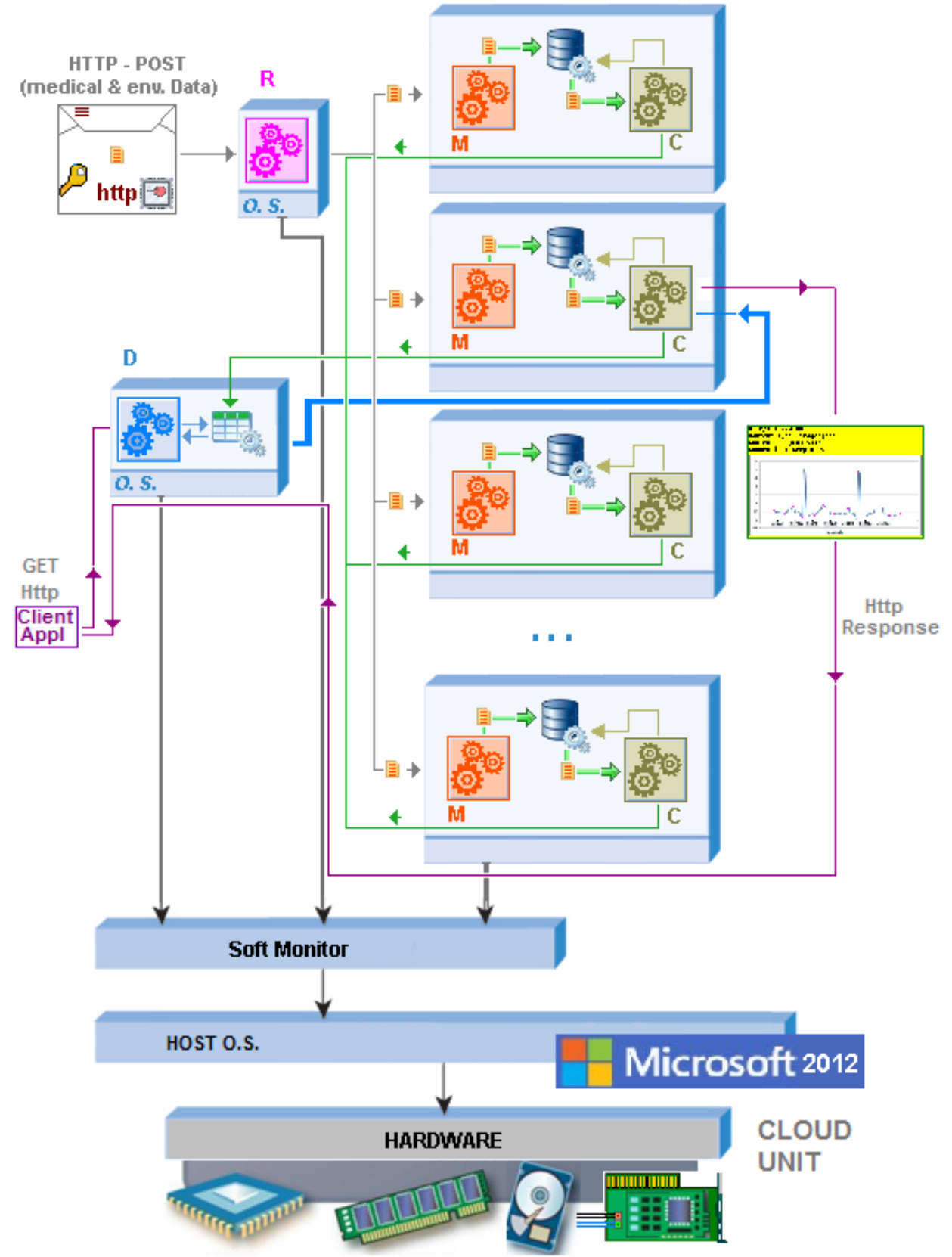

Figure 2. Optimization of request management based on Services distributed on Multiple VM

The server component (server-side) of the system consists of the following subcomponents:

- (R:1) The service that is intended to receive and organize into the storage space all sent data. It works inside its own virtual machine. The primary role of this service is to insure for the actual data (extracted from the incoming HTTP-Post request) a simultaneous storage in multiple identical databases, each base running in its own virtual machine. The 
service immediately redirects ("broadcasts") current data to the ensemble of services introduced immediately below. The duplication process of medical data storage has a dual role:

- It allows balancing the processing (computing) effort in order to maintain more or less the same response readiness in the event of a heavy flow of requests issued by various client applications;

○ It provides an important safety enhancement for data storage.

- (M:n) Data memorizing (storage) services have the role of writing the data received from the patient in an associated database. Each service works in conjunction with a NoSQL database management system. Each service works inside its own virtual machine.

- (D:1) The dispatch service also works inside its own virtual machine. It captures and manages data processing requests. The role of this service is to assess the distribution of the current computing effort over the actual computing ensemble and to select the most appropriate computing unit (i.e. the most "free") that would be entrusted with resolving the current request. When a request is sent to a certain computing unit, this dispatch service promptly updates to an associated database the loading status of the machine (the computing unit) to which the current request has just been sent.

- (C:n) Services of computational unit type. Each of them serves three main purposes:

$\circ$ reads appropriate data (pertaining to the current request) from the associated database.

○ processes retrieved data and send the response to the requesting client application.

0 instantly after sending the response to the requesting client application, it notifies the dispatcher (through a Http-PUT request) regarding its newly established availability. A single "C" type service and a single "M" type service are hosted by the same Virtual Machine.

It must be stated that every alphanumerical symbol (following the colon, inside de brackets that open each main enumeration of services) indicates the multiplicity of the service(s) within the architecture of the solution (i.e. "1" indicates that a certain service has a single instance, whereas " $n$ " suggests that the introduced service is configured to have multiple instances).

\section{Conclusion}

Health monitoring of the seniors in an IoT-enabling smart environment has the capacity to provide personalized services for a qualitative life of the elderly in their homes. The huge amount of healthcare data gathered inside this environment have particular features like heterogeneity, privacy, time-sensitivity. In order to be efficiently stored, analyzed and processed to get the most appropriate insight and applications, an optimized management of healthcare data should be performed.

The integrated approach of IoT and Cloud technologies in the monitoring of physiological and environmental parameters as well as the development of predictive models for population health allow the development of innovative personalized assistance services.

In the context of the ongoing RO-SmartAgeing project, a proposed server-side architecture was presented aiming at optimizing the response speed (that is reducing processing time and shortening waiting intervals) in the most likely scenario of a heavy flow of requests issued by various client applications.

Balancing the computing load is achieved by the means of using an ensemble of computing services, each one running under its own virtual machine. Re-distribution of incoming requests is performed at extremely high speed between virtual machines belonging to the same Cloud Unit. 
www.conferenceie.ase.ro

\section{Acknowledgment}

This work was supported from the project "Non-invasive monitoring and health assessment of the elderly in a smart environment (RO-Smart Ageing)" (funded by the Romanian Core Program of the Ministry of Research and Innovation) (2018-2020).

\section{References}

[1] Eurostat, Population structure and ageing, Available: https://ec. europa.eu/eurostat/statis ticsexplained/index.php?title=Population_structure_and_ageing\&oldid=423053\#Past_and_ future_population_ageing_trends_in_the_EU, May 2018 [April, 2019].

[2] National Institute of Aging, World Health Organization, "Global Health and Aging", October 2011, pp. 8. Available: https://www.nia.nih.gov/research/publication/globalhealth-and-aging/ humanitys-aging.

[3] S. Vitabile, et al., "Medical Data Processing and Analysis for Remote Health and Activities Monitoring”, in: Kołodziej J., González-Vélez H. (eds) High-Performance Modelling and Simulation for Big Data Applications. Lecture Notes in Computer Science, vol 11400. Springer, Cham, 2019, pp 186-221.

[4] M. Ianculescu, A. Alexandru and E. Tudora, "Opportunities brought by big data in providing silver digital patients with ICT-based services that support independent living and lifelong learning”. In: Proc. 2017 Ninth International Conference on Ubiquitous and Future Networks (ICUFN). IEEE, 2017. pp. 404-409.

[5] M. M. Dhanvijay and S. C. Patil, "Internet of Things: A survey of enabling technologies in healthcare and its applications", Computer Networks, 2019.

[6] M. Ebling, "Pervasive Computing and the Internet of Things”, In „IEEE Pervasive Comput., pp. 2-4, 2016.

[7] M. Kocakulak and I. Butun, "An overview of Wireless Sensor Networks towards internet of things", In Proc. 2017 IEEE 7th Annual Computing and Communication Workshop and Conference (CCWC) 2017 Jan 9, p. 1-6, IEEE. 2017.

[8] J.T. Erber, Ageing and Older Adulthood, third ed. Wiley-Blackwell, Chichester, 2013.

[9] H. Li, J. Wu, Y. Gao and Y. Shi, "Examining individuals' adoption of healthcare wearable devices: An empirical study from privacy calculus perspective", International journal of medical informatics, Vol. 88, pp 8-17 Apr 2016.

[10] S.T. Rosenbloom, "Person-generated health and wellness data for health care", J. Am. Med. Inf. Assoc., Vol. 23, Issue 3, pp. 438-439, 2016.

[11] H. Elazhary, "Internet of Things (IoT), mobile cloud, cloudlet, mobile IoT, IoT cloud, fog, mobile edge, and edge emerging computing paradigms: Disambiguation and research directions", Journal of Network and Computer Applications, 2018.

[12] A. Mosa, I. Yoo and L. Sheets, "A systematic review of healthcare applications for smartphones”, BMC Med. Info. Decis. Making, Vol. 12, Issue 67, 2012.

[13] Actus Digital. Virtual Machine or Physical Server. Available: https://actusdigital.com/virtual-machine-vs-physical-server/, January 2018 [April, 2019]. 